\title{
Web Image Re-ranking using Semantic Signature and Visual Query Expansion
}

\author{
Khushabu A. Surana \\ PG Student \\ Department of Computer Engineering , \\ SSBT's college of engineering \& Technology \\ Bambhori,Jalgaon.
}

\author{
Shital Patil \\ Assistant Professor \\ Department of Computer Engineering , \\ SSBT's college of engineering \& Technology \\ Bambhori,Jalgaon.
}

\begin{abstract}
Web mining is a important aspect for users to get accurate and relevant data as per user need and satisfaction. Web image reranking is an effective way to improve the results of web based image searching. A major challenge in web image reranking is that the similarities of visual features are not correlated with images and semantic meanings which can not interpret users search intention. In proposed approach, By providing keyword expansion to textual query and visual query expansion to image query the results retrieved are promising as compared to existing system. Feature extraction, Hashing, Euclidean distance are used to make semantic signature more compact and to further enhance efficiency.
\end{abstract}

\section{Keywords}

Web image re-ranking, Semantic Signature, Visual Query Expansion, Hashing

\section{INTRODUCTION}

Data mining, also called as knowledge discovery from database (KDD). It is the branch of computer science. In the past few decades, advances in data mining techniques lead to many remarkable revolutions in big data. Data Mining (DM) is the process of discovering hidden data from data warehouse, develop the model and discover previously unknown patterns. A major challenge for next generation of data mining system is to discovery related data from highly different data, distributed data and knowledge sources. The effectiveness of domain knowledge in data mining has been attested in past research efforts. Data mining software is just like analytical tools for analyzing data. It allows user to analyze data from the different view, categories and dimension.

\subsection{Overview of Data Mining}

Data mining is the process of extraction of implicit, previously unknown, and useful information from data. Data mining is a task in the process of knowledge discovery. Data mining is a core part of KDD process. Knowledge Discovery in Databases (KDD) is an automatic and modelling of large data repositories. KDD is the process of finding valid, novel, useful, and understandable patterns from large and complex data sets. It is of interest to researchers in machine learning, pattern recognition, databases, statistics, artificial intelligence, knowledge acquisition for expert systems, and data visualization. The KDD process consist of Data selection, Data cleaning, Data transformation, Pattern matching, Finding presentation, Finding interpretation, and Finding evaluation. Data mining and KDD many times used interchangeably because data mining is the key part of the KDD process. Overview of typical KDD process is shown below in Figure 1.1. The overall process of finding and interpreting patterns from data involves the repeated application of the following steps:

- Development of an understandable and desirable application domain.

- Perform discovery process for which Selecting and creating a data set from the data warehouse.

- Pre-processing and enhancing the reliability of data.

- Data transformation in which generation of better data for data mining is prepared.

- Choosing the Data Mining task like clustering, classification, regression etc.

- Choosing the appropriate Data Mining algorithm.

- Implementing the Data Mining algorithm.

- Evaluation of interpreted and mined patterns.

- Deployment using discovered knowledge.

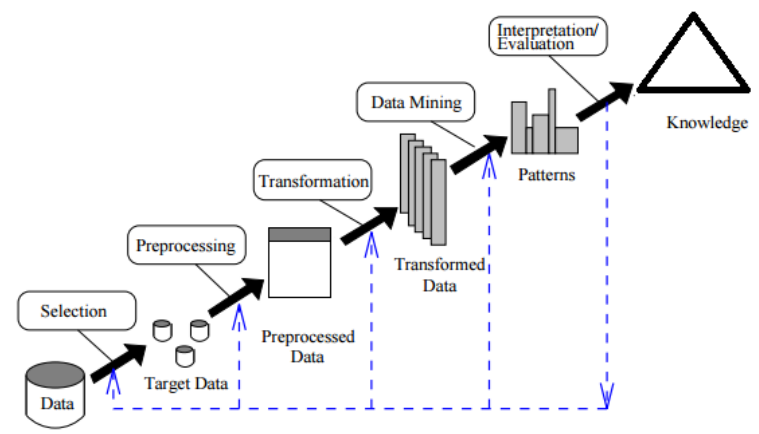

Figure 1.1 Overview of KDD Process

The process of finding and interpreting patterns from data begins with application domain and stops on implementation of the discovered knowledge. Then the loop is stoped and actual data mining part begins the process. After the mining the changes can be done in application domain. Discovered knowledge stops the loop, effect are measured on new data and KDD process starts again. Data mining means to understand, analyze and eventually use of such large data as a multidisciplinary approach. There are several data mining techniques such as association, classification, clustering, prediction, sequential patterns and decision tree. All those data mining techniques are briefly mentioned below:

- Association: Association is one of the best data mining technique. In this type of mining data is arrange based on relationship between items. The 
items should be from same transaction. That's why association technique is also known as relation technique. The association technique is used in market basket analysis. Using this technique customer can identify a set of products that used frequently purchase together.

- Classification: Classification is a classic data mining technique based on machine learning. This is used to classify each item in a set of data. This method makes use of mathematical techniques such as decision trees, linear programming, neural network and statistics. In this method, the software learns how to classify the data items into groups. In the classification techniques, objects are assigned into predefined classes.

- Clustering: Clustering is one of the data mining technique in which different cluster are makes using various technique. Cluster is the collection of different objects. Clustering technique defines the classes and puts objects in each class. In this technique, related data is grouped into multiple cluster from the large amount of data.

- Prediction: The prediction is one of a data mining techniques that discover relationship between independent variables and relationship between dependent and independent variables. For instance, the prediction analysis technique can be used in stock market to predict profit for the future.

- Frequent Patterns: Frequent patterns are patterns that appear in a data set frequently. Frequent patterns analysis is one of data mining technique that gets to discover or identify similar patterns, regular events or trends in transaction data over a business period. frequent pattern mining searches for recurring relationships in a given data sets.

\subsection{Overview of Web Mining}

Web mining is most popular among research today. Web mining is the technique of data mining which used to discover and extract information from web document and services. Then the extracted information from web documents or services and can be applied to semi-structured or unstructured data like free-from texts. Mining the useful data from the web using various data mining techniques and tools is called web mining. It is most important application of data mining and other information processing technique for finding useful patterns of data. It describes the application of traditional data mining techniques onto web resources. It is used to understand customer behaviour, evaluation of effectiveness of particular web site. Web mining can be divided or classified into three categories:

- Web Content Mining (WCM):- Web content mining is the process of discovery and extraction of useful information. Web content mining, also known as text mining. The content of the raw data are available on the one hand, and the expected knowledge to be derived from it. It is generally the second step in Web data mining. Content mining is the scanning and mining of text, pictures and graphs from the Web page. Which is use to determine the related data of the content to the search query. This scanning is completed after the clustering of web pages through structure mining. It gives the results based upon the level of relevance to the suggested query. With the massive amount of information that is available on the World Wide Web, content mining gives the results lists to search engines in order of highest relevance to the keywords in the query.

- Web Structure Mining (WSM):- Web structure mining is one of the categories of web mining. It is related to analyzing hyperlink and link structure on web for information retrieval and knowledge discovery of any type of data or items. It is specially use to extract previously unknown relationship between web pages. Structure mining is use to minimize the problem of World Wide Web due to its huge amount of data. This kind of mining is use to determine the similar structure from the web. Web structure mining is specially use in business word.

- $\quad$ Web Usage Mining (WUM):- Web usage mining is third category of the data mining technique which is use to discover interesting useful pattern from web data in order to understand and better serve the needs of web based applications. It is the process of extracting information from user data history from web, either textual, or multimedia. Usage data captures the identity or source of web users along with their browsing behaviour at web site. Usage mining allows companies to produce productive information related to the future of their business function ability.

\subsection{Motivation}

A process in which images are retrieved and arranged as per their features and user need is called web image re-ranking. To facilitate access to the rapidly growing collections of images on the Web and maximize their benefit for the users, image search has become an increasingly important research topic. An image search engine generally operates in two main steps: the offline index generation and the online index serving step. Meaningful Image retrieval is a challenge for effective web search. By using existing technique ambiguity is occurred in image retrieval. Sometime accurately describing the visual content of target images only using keyword is difficult for user. Due to lack of query and visual features images obtained are less relevant as per user need. To overcome drawback of existing system semantic signature and visual query expansion plays an important role in web image re-ranking.

\section{LITERATURE SURVEY}

In the past, there was lot of research done on the image retrieval. The huge number of available images on the web makes image retrieval a challenging task. There are various techniques used by image retrieval to retrieve the most relevant image.

\subsection{Background}

There are so many techniques that have been put forward for better performance of web image re-ranking in the recent days. Web image re-ranking, is a mixed process of both text based image result and visual features to obtained good performance in image search. In this process text query is given by the user and the search is done. It gives the mismatched images which are not related to query and not useful to user. After that query image is selected by user and again the search will be done. Then user get the most relevant image based result, after that re-ranking is done. 
Most existing methods rely on the same framework: I. The text query is used to retrieve a noisy set of images using a text-based search engine, II. In some approaches, this initial set of images is filtered by removing drawings and other nonphotographic images, then III. A classifier, specific to the given query, is learned from this image set. For example, using "Apple" as a query keyword, the retrieved images belong to different three main categories, such as "Green Apple" (Fruit), "Apple iphone" (Mobile), and "Apple" (Laptop). Within each main class, there can be several images that are visually similar. Also, there are images that can be irrelevant images or hard to judge relevancy.

There are three approaches using which images are retrieved. I. Text based approach in which images are retrieved based on text. II. Content based approach in which images are retrieved based on content. III. Hybrid approach in which images are retrieved based on both content and text. Recently so many methods have been proposed for image search re-ranking, which can be divided into three categories: classificationbased, clustering-based, graph based. Several works have done in web image re-ranking.

\subsection{Related Work}

Krapac et al.,[1], this paper introduced the generic classifiers based on query-relative features which could be used for new query keywords without additional training. But the problem that, this is unsuitable for real world web search applications. The Main aim of image re-ranking is to find out visual similarities of images and re-ranking the remaining images. Re-ranking is based on their visual similarity. Which are reflecting semantic relevance of images for different query images.

Cui et al.,[2],[3] has classified query images into eight predefined intention categories. This gives the different feature weighting schemes to different types of query images. But it is difficult for eight weighting schemes to cover the large diversity of all the web images. And it is also do the wrong classification using query image. $J$ cui gives the Real time Google, live image search re-ranking and Intent search: Interactive online image search re-ranking

Duan et al.,[4], this paper represents the bag-based re-ranking method. This method is proposed for large-scale text based image search. In this method firstly textual query is given to text based image retrieval and then related images are display. Then images are re-ranked after the text based image search. The relevant images are clustered using both textual and visual features. By treating that each cluster as a bag and images in the as instances. Advantage is that the bad based method can achieve the best performance. Disadvantage is that it ambiguity may occur in the labels of instances.

Tang et al.,[5], in this paper the intent search of images is proposed. In this method of image retrieval and re-ranking, user should click on one query image with minimum efforts . Images are retrieved from a pool by text-based search are reranked based on both visual and textual content. The main contribution is to capture the users search intention from this one-click query image. Advantage is that the user will get more relevant images based on clicked query images. Disadvantage is that more word is added for labelling the regions.

Gordon et al.,[6], in this paper by applying the information bottleneck principle to unsupervised clustering of discrete and continuous image representation. The unsupervised re-ranking methods do not rely on human labelling of relevant images but require prior assumptions on how to employ the information contained in underlying text based result for reranking.

Baxi et al.,[7], represents instead of using multiple clusters, re-ranking can be simplified using binary classification. There are normally three steps: select the pseudo-positive and pseudo-negative samples from the initial text-based search results; train a classifier using the selected samples; and reorder the samples according to the relevance scores predicted by the trained classifier. In the first step pseudo relevance feedback is used to select the training samples. This concept is introduced from the text retrieval. In the second step various classifier are used.

Sunitha et al.,[8], the intent search re-ranking methods may fail to retrieve the user's intention when the given query term is ambiguous. So the re-ranking with user interactions, known as active re-ranking is very effective. It improves the web image search results. One of the main problems in active reranking is how to find the user's intention. For this structural information based sample selection strategy is used. It minimizes the labelling efforts of the users.

Tian et al.,[9], this paper gives visual information of images. This is insufficient to infer the user's intention, when the query term is ambiguous. The re-ranking with user interaction is proposed in which some images are selected according to an active sample selection strategy, and then user is required to label them. After that the re-ranking process is conducted with the help of labelling information and learned properties.

Yang et al.,[10], in this paper the previous methods for image search re-ranking suffer from the unreliability of the assumptions under which the initial text based image search result is employed in the re-ranking process such as labelling of samples by user. A prototype-based re-ranking method is proposed to address previous problem in a supervised, but scalable fashion. By applying different meta re-rankers to an image from the initial result, re-ranking scores are generated, which are then aggregated using a linear model to produce the final relevance score and the new rank position for an image in the re-ranked search result.

Pushpaltha et al.,[11],has given instead of labelling, user click information can been used in image re-ranking, because clicks have been shown to more accurately describe the relevance of retrieved images to search queries. However, a critical problem for click-based methods is the lack of click data, since only a small number of web images have actually been clicked on by users. Therefore, for predicting image clicks multimodal hyper graph learning-based sparse coding method is proposed, and applied the obtained click data to the reranking of images.

\section{PROPOSED SYSTEM}

The proposed approach describes the solution to image retrieval. An existing system consists of lack of image retrieval and proposed solution overcomes this drawback by using visual query expansion and semantic signature. An accurate and most relevant image retrieval is possible only when some technique are said to exist. Visual query expansion is provided using hashing, feature extraction and keyword query expansion provided using synonyms. Visual query expansion and semantic signature is best for such task. Features are extracted at the time if images inserted into database. Keyword expansion and visual expansion act as a technique to retrieval of most relevant image and re-rank other images based on their Euclidean Distances. In this way 
most relevant image is retrieved which is hidden in the database. Use of semantic signature and visual query expansion as a web mining technique in various data mining techniques found effective for improving the precision of image retrieval.

\subsection{Proposed System Architecture}

Figure 1.2 shows architecture of the proposed system. There are two stages: offline stage and online stage. features are extracted at the time of image insertion in database. At the offline stage when user put text query in query box then semantic signature of that query image is calculated and store in database. Most of the work is done at the offline stage. At the online stage, the user receives the final result after the semantic signature and visual query expansion is calculated at offline stage. Keyword expansion gives the synonyms of the text query given by user. After the text based result user select the query image and then visual query expansion is find out at offline stage and re-ranking is shown at online stage.

\subsubsection{Feature Extraction}

In the proposed system, colour features are extracted at the time of image insertion in database. Firstly images are converted into gray scale. After converting the image into gray scale mean is calculated for each image. By using the value of mean, standard deviation is find out. Mean and standard deviation is stored in the visual feature database table. And that database value used at the time of image comparisons.

\subsubsection{Keyword Expansion}

Keyword expansion is use to target new keyword by exploring various synonyms that are both relevant and traffic driving. Keyword1 generate the synonyms which are given by user at the time of image insertion. Then keyword2 is use to find the synonyms through WordNet. Based on the keyword1 and keyword2 images are retrieved for the further process. The keywords given by user are some time short. They cannot gives the content of images accurately. The query keywords meanings may be richer than user's expectations. For example, the meanings of the word flower include rose, lotus, and sunflower etc. The user may not have enough knowledge on the textual description of target images. Set of keyword Expansions define reference classes for different keyword.

\subsubsection{Visual Query Expansion}

Visual query expansion includes the multiple positive example images to learn a visual similarity. When 0user select the query image then visually similar images are retrieved. There are 10 type of classes in the database which are inserted after applying the feature extraction. Here, user select one image from the keyword based image result and based on that one image four images are retrieved using feature extraction in visual query expansion. The distance between that images are nearly similar. The chosen image cluster have the closest visual distance with the user selected query image. So that user can get the most accurate and relevant result.

\subsubsection{Classifier of reference classes}

SVM is adopted to improve the visual similarity. This takes the re-ranked image as input to the one-class SVM classifier and similarity to the query image is uses as output. user give the class name at the time of images insertion in database. SVM is a supervised algorithm which is used to classify the issues. It uses the different algorithms to transform the data.

\subsubsection{Semantic Signature over reference classes}

It is necessary to discover semantic features for each image. Semantic feature discovery based on multiple modalities such as image contents and tags. Using semantic signature over reference classes user get the appropriate intention image. Reference classes give the different categories for image search to get the most accurate image.

\subsubsection{Hashing}

Hashing means converting the image into gray scale and getting the hash value to perform hamming distance. Hashes are generated from the input using extracted features that display invariance to common intra-class variations. In hashing firstly feature from image is extracted. Then the second stage compresses this feature vector to a final hash value. In this paper perceptual algorithm is used to convert image into gray scale and resize the given image.

Perceptual hashing is a robust algorithm widely used for content identification. P-hash is reliable and fastest algorithm. Perceptual image hashing is conventionally used for content identification and authentication. The hash string is generated using the colour of an images. Pixel colour average is taken as mean value based on the average rate the hash string is formed. The image is scaled to defined small size. After the generation of the hash value, colour pixel and its average colour pixel value. Hash bit is generated, with respect to the average of total colour pixels and each colour pixels of the image.

\subsubsection{Euclidean Distance}

The Euclidean distance is the straight-line distance between two pixels. It is the distance between two points in euclidean space. The two points A and B in two dimensional euclidean spaces and A with the coordinates (a1, a2), B with the coordinates (b1, b2). The line segment with the endpoints of $\mathrm{A}$ and $\mathrm{B}$ will form the hypotenuse of a right angled triangle. The distance between two points $\mathrm{A}$ and $\mathrm{B}$ is defined as the square root of the sum of the squares of the differences between the corresponding coordinates of the points. The Euclidean distance between two points is:

$\mathrm{D}(\mathrm{A}, \mathrm{B})=\sqrt{ }(\mathrm{b} 1-\mathrm{a} 1)^{\wedge} 2+(\mathrm{b} 2-\mathrm{a} 2)^{\wedge} 2$

\subsubsection{Re-ranking}

At the online stage, after finding euclidean distance

the images are re-ranked. A process in which images are retrieved and arranged as per their features and user need is called web image re-ranking. The re-ranking of the returned images based on metadata of image and text surrounding of image alone. Extend and follow the method proposed by using a set of textual features which presence is great indication of the image content. The aim is to re-rank the searched images. Each feature is used as binary: "True" if it contains the query

text and "False" otherwise. To re-rank images for one fix class, we do not employ the whole images for class.

\subsection{Algorithm and Steps}

1. Query keyword is entered by the user. And features are extracted at the time of image inserted in dataset.

2. With the help of Wordnet Dictionary, keyword expansion of query is been done. Along with semantic keywords are retrieved for the specified query. 
3. Image results are been generated according to the query specified and its semantic, hypernonym, hyponyms of keywords are used.

4. User selects a query image from the generated output.

5. Image expansion is done to find similar images with count of 4.

6. Histogram is calculated or all images retrieved from image expansion and all the images of dataset.
7. Perceptual Hashing is applied on the histogram output of retrieved images and dataset images.

8. Finally Euclidean distance is calculated for each retrieved image from image expansion with all images from dataset.

9. If the Euclidean distance is within particular range the final queries are displayed as final output.

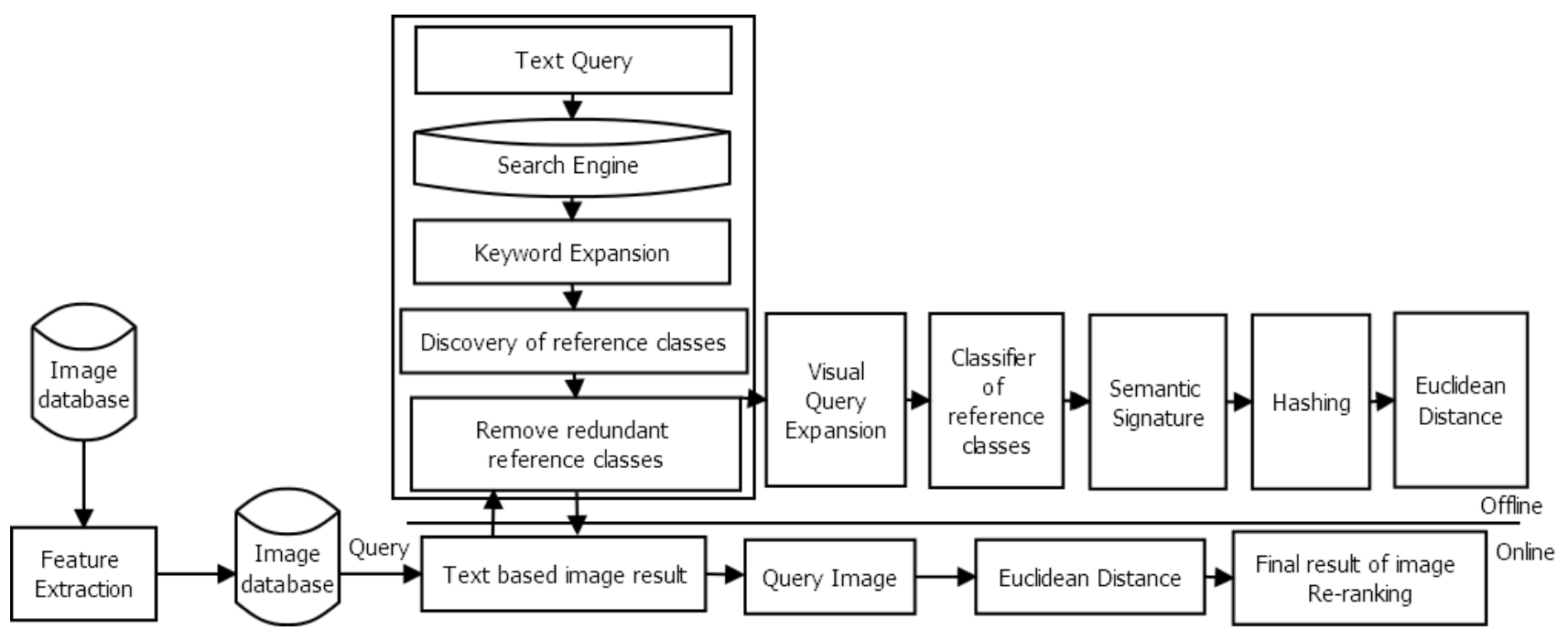

Figure 1.2 : System Architecture of Proposed

\section{RESULT AND DISCUSSION}

The proposed system is evaluated via experiments on the real data. The real world data is provided as input to the proposed system in a specific format. It is applied as it is available to the proposed system. The proposed system is tested on the image dataset. Dataset contains 2000 images with number of classes. Image dataset is divided into number of classes and that particular class contains the number of images. Feature can be extracted or calculated at the time of image inserted in dataset. After image re-ranking precision, recall and Fmeasure is calculated. Precision and recall are the basic measures used in evaluating search strategies.

- Precision:- Precision is the basic measure used in evaluating search strategies. Precision means how many selected items are relevant?

- Recall:- Recall is the ratio of the number of relevant records retrieved to the total number of relevant records in the database. It is usually expressed as a percentage. Recall means how many relevant items are selected?

- F-measure:- F-measure is calculated from precision and recall.

Following Table1 shows the average percentage of Precision, R0ecall and F-measure. Initially flower class is taken in consideration which gives the $72 \%$ precision, $66 \%$ recall, $68.86956522 \% \mathrm{~F}-\mathrm{measure}$. Ball class is taken in consideration which gives $69 \%$ precision, $65 \%$ recall, $66.94029851 \%$ Fmeasure. Car class is taken in consideration which gives $66 \%$ precision, $62 \%$ recall and $63.9375 \%$ F-measure. Forest class is taken in consideration which gives $68 \%$ precision, $59 \%$ recall and $63.18110236 \%$ F-measure. Mountain class is taken in consideration which gives $72 \%$ precision, $68 \%$ recall and
69.94285714 \% F-measure etc. Similarly Apple, Bat, Buttery, Palm classes are taken in consideration. Figure 1.3 shows the bar graph in which precision is represented at y axis and class name is represented at $\mathrm{x}$ axis. Figure 1.4 shows the bar graph in which recall is represented at y axis and class name is represented at $\mathrm{x}$ axis. Figure 1.5 shows the bar graph in which F-measure is represented at y axis and class name is represented at $\mathrm{x}$ axis. Figure 1.6 shows the bar graph in which comparison of precision, recall and F-measure is represented at $\mathrm{y}$ axis and class name is represented at $\mathrm{x}$ axis.

The images retrieval is the integral part of the data mining and web mining. The performance of the proposed system is evaluated through the real world data set. The objectives of the proposed system was identification of the user interested image item sets. Groundtruth image dataset are used to represent user interest in web mining. By using semantic signature and visual query expansion, The proposed system generate the user interested image from dataset.

Table 1. Dataset for all classes

\begin{tabular}{|l|c|c|c|}
\hline $\begin{array}{c}\text { Class } \\
\text { name }\end{array}$ & Precision & Recall & F-measure \\
\hline Flower & 72 & 66 & $68: 86956522$ \\
\hline Ball & 69 & 65 & $66: 94029851$ \\
\hline Car & 66 & 62 & $63: 9375$ \\
\hline Forest & 68 & 59 & $63: 18110236$ \\
\hline Mountain & 72 & 68 & $69: 94285714$ \\
\hline Apple & 62 & 56 & $58: 84745763$ \\
\hline
\end{tabular}




\begin{tabular}{|l|c|c|c|}
\hline Bat & 58 & 55 & $56: 4601769$ \\
\hline Butterfly & 68 & 72 & $69: 94285714$ \\
\hline Palm & 58 & 54 & $55: 92857143$ \\
\hline
\end{tabular}

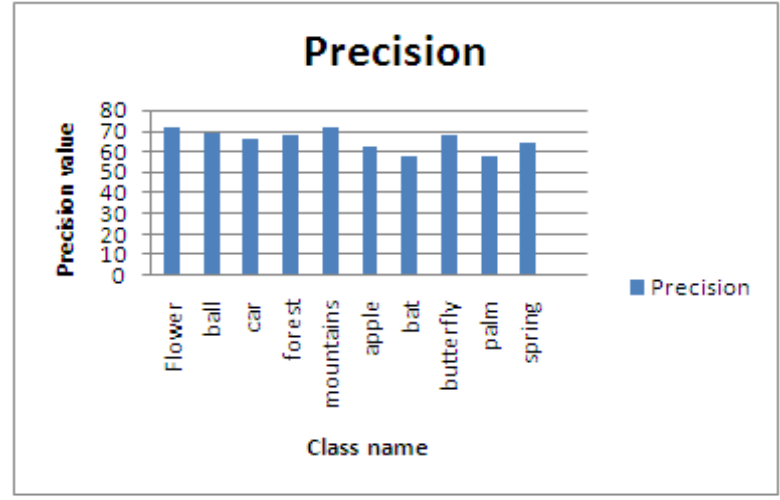

Figure 1.3 : Bar graph representation of Precision.

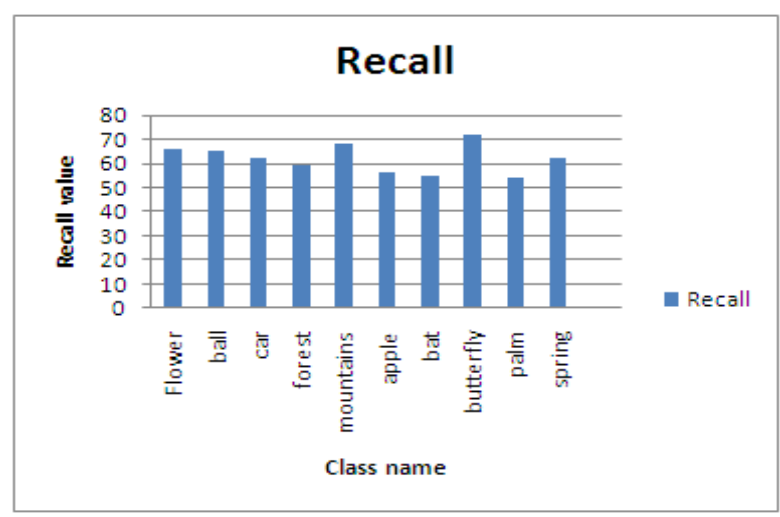

Figure1.4 : Bar graph representation of recall.

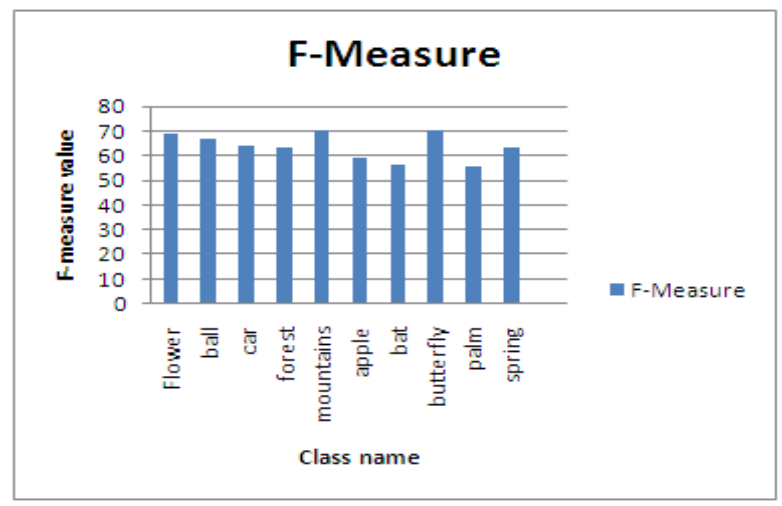

Figure 1.5 : Bar graph representation of F-measure.

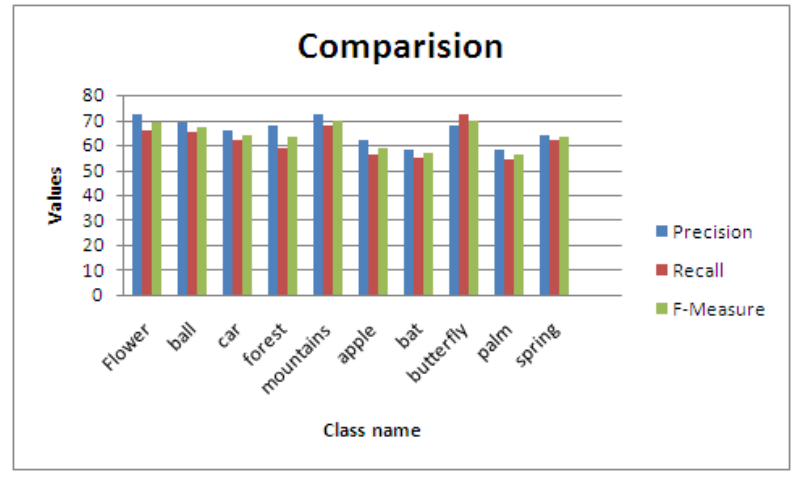

Figure1. 6 : Combine bar graph representation of precision, recall, F-measure.

\subsection{Feature Extraction after image inserted in dataset}

In the proposed system, images are inserted in dataset and then process is begin. Keyword expansion gives

the synonyms of the query keyword. keyword1 gives the synonyms of user inserted as the time of image insertion. Keyword2 gives the synonyms through Wordnet. After that Visual query expansion is use to select the four query image. At the time of visual query expansion Hashing, feature extraction, DCT, Histogram, Euclidean distance is find out. Images are arrange in ascending order which depends on the euclidean distance. Re-ranking is done after arranging the images in the ascending order. And user select the top ranked images which are in the same semantic category as the query image. Visually similar to query image should have higher rank. In this way of proposed work take the more time to rerank because features are extracted after the image inserted in dataset. Duplication is also occurs after the text based image result. This re-ranking process is some time complex in nature.

\subsection{Feature Extraction at the time of image inserted in dataset}

In the proposed system, images are inserted in dataset and same time features are extracted, then process is begin. Keyword expansion gives the synonyms of the query keyword. keyword 1 gives the synonyms of user inserted as the time of image insertion. Keyword2 gives the synonyms through Wordnet. After that Visual query expansion is use to select the four query image. At the time of visual query expansion Hashing, DCT, Histogram, Euclidean distance is find out. Images are arrange in ascending order which depends on the euclidean distance. Re-ranking is done after arranging the images in the ascending order. And user select the top ranked images which are in the same semantic category as the query image. Visually similar to query image should have higher rank.

In this way of proposed work advantages are, this process take the less time to re-rank because features are extracted at the time of image inserted in dataset. so that it consumes the time which user require for further enhancement of process. Duplication is also avoided after the text based image result. This re-ranking process is not complex in nature. This process gives the most relevant and accurate images. By using this method image re-ranking become extremely efficient. And computational class is also reduced by using this method of re-ranking. 


\section{CONCLUSION}

Image re-ranking is the process of retrieving more accurate and relevant images from the dataset. In traditional approach, most of the images remain hidden in the dataset itself due to lack of text and content knowledge. Thus experiments proved that in proposed re-ranking framework, user learns semantic signature to significantly improve the effectiveness and efficiency of image re-ranking. The visual features extraction of images projected in the proposed system has shown improved and better accuracy of image re-ranking.

Also Experiments shows the extracted semantic signatures can be 70 times shorter than the original visual feature on average, while proposed work achieve $20 \%$ upto $35 \%$ relative improvement on re-ranking over existing system using performance parameters like precision, recall and F-measure.

In Future Research, consideration of time complexity would be important point to focus. Also various algorithms can be merged with proposed solution to test and increase accuracy of image re-ranking. Proposed system can be applied on real world dataset to test the accuracy of proposed system.

\section{REFERENCES}

[1] J. Krapacy, M. Allanyy, J. Verbeeky, and F. Juriey, "Improving web image search results using queryrelative classifiers," IEEE Transaction, vol. 31, 2011.

[2] J. Cui, F. Wen, and X. Tang, "Real time google and live image search re-ranking,"Journal of Computing, October 2008.

[3] J. Cui, F. Wen, , and X. Tang, "Intent search: Interactive online image search rerank-ing," 16th ACM Intel Conference Multimedia, October.

[4] L. Duan, W. Li, I. W.-H. Tsang, and D. Xu, "Improving web image search by bag-based re-ranking," IEEE
Transactions On Image Processing, vol. 20, no. 11, pp. 3280-3290, November 2011.

[5] X. Tang, K. Liu, J. Cui, and F. Wen, "Intentsearch: Capturing user intention for one-click internet image search," IEEE Transaction on Pattern Analysis and Machine Intelligence, vol. 34, no. 7, pp. 1324-1353, July 2012.

[6] S. Gordon, H. Greenspan, and J. Goldberger, "Applying the information bottleneck principle to unsupervised clustering of discrete and continuous image representations," IEEE International Conference on Computer Vision, vol. 2, 2003.

[7] S. Baxi and S.V.Dabhade, "Re-ranking of images using semantic signatures with duplicate images removal and k-means clustering," International Journal Of Engineering And Computer Science, vol. 3, no. 5, pp. 6122-6127, May 2014

[8] S.Sunitha and A. RamaSatish, "Extended image features for user intention refined image search," International Journal of Advanced Research in Computer Science and Software Engineering, vol. 4, February 2014.

[9] X. Tian, D. Tao, X.-S. Hua, and X. Wu, "Active reranking for web image search," IEEE Transactions On Image Processing, vol. 19, pp. 805-820, March 2010.

[10] L. Yang, A. Hanjalic, and S. Member, "Prototype-based image search re-ranking," IEEE Transaction on Multimedia, vol. 14, no. 3, June 2012.

[11] N. Pushpaltha and G. P. Reddy, "Multimodel sparse code for re-ranking of web image prediction," International Journal of advance research in Computer science, vol. 3 , no. 4, April 2015 